\title{
Postpartum cardiac hemodynamics after normal delivery, preeclampsia and eclampsia: a prospective study
}

SECHENOV

UNIVERSITY

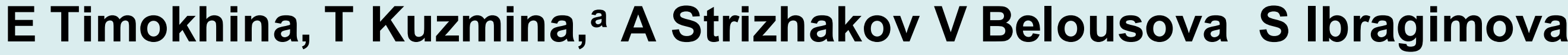

The objective The aim of this study is to assess maternal hemodynamics immediately in the postpartum period, and also after 2 and 6 months, in the parturients who were diagnosed with preeclampsia and eclampsia and to evaluate the long- term risks of developing heart dysfunction, heart failure, and vascular diseases.

\section{Design Prospective observational case-control study}

Population 90 postpartum women after mild and severe preeclampsia and eclampsia (main group) and 55 patients after an uncomplicated pregnancy (control group). The parameters of maternal hemodynamics were recorded on $1,3,5,9,14$ day postpartum period, as well as after 2 and 6 months after delivery. Indicators of the control group compared with the hemodynamic parameters in nonpregnant patients of reproductive age without somatic diseases (30 cases).

Methods The following indicators were assessed by echocardiography: end-systolic volume (ESV), end-diastolic (EDV), stroke volume (SV), cardiac output (CO), stroke index (SI) and cardiac index $(\mathrm{Cl})$, mean velocity of circumferential fiber shortening (MVCF), heart rate (HR), mean blood pressure (MBP) and systemic vascular resistance (SVR).

\section{Cardiac parameters}

Our studies have shown that women with mild and severe of preeclampsia in the 1-3 days after delivery have shown disorders of the cardiac hemodynamics, manifested in significantly lower values of volume indicators and the contractility of the myocardium with increased in mean blood pressure and systemic vascular resistance. Later we saw restoration of blood circulation parameters, with the rate of improvement depending on the severity of PE.
Thus, in the mild PE significant increase in EDV, SV and SI was observed on third day postpartum, peripheral vascular resistance (PVR) decreased - on the 5th day of postpartum period, but the normalization of these parameters is seen only by $12-14$ days after labor.

The maternal hemodynamics of the puerperas whose pregnancies were complicated by $P E$ and $E$ is characterized by impaired contractility of the myocardium and an increased in the indices of peripheral vascular resistance. The degree of deviation in the parameters of cardiac hemodynamics and vascular resistance depended on the severity of hypertensive complications of pregnancy. Patients after PE / E are at higher risk of long-term cardiovascular disease and require cardiologic follow up and tight control of blood pressure.

Keywords preeclampsia, eclampsia, maternal hemodynamics, gestational hypertension, , echocardiography, ophtalmic arteries, renal arteries, Doppler parameters

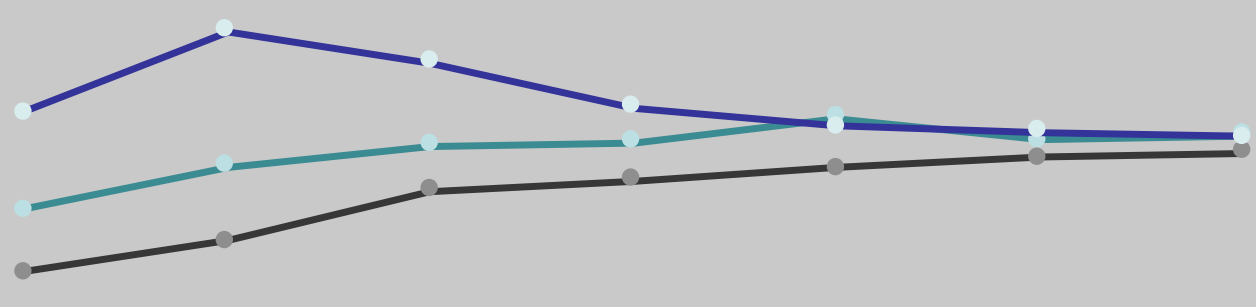

\begin{tabular}{|c|c|c|c|c|c|c|c|c|}
\hline \multirow{2}{*}{$\begin{array}{c}\text { Hemodynamic } \\
\text { parameters }\end{array}$} & & \multicolumn{7}{|c|}{ Period after labor } \\
\hline & & 1 day & 3 days & 5 days & 9 days & 14 days & 2 months & 6 months \\
\hline \multirow[t]{3}{*}{ Stroke volume (SV), ml } & $\begin{array}{c}\text { Mild PE } \\
(n=39)\end{array}$ & $52,57 \pm 2,01^{*}$ & $60,62 \pm 2,01 *$ & $64,24 \pm 2,61 * *$ & $65,02 \pm 2,11 * *$ & $69,18 \pm 2,03$ & $65,18 \pm 2,61$ & $66,29 \pm 2,34$ \\
\hline & $\begin{array}{c}\text { Severe } \\
\text { PE/eclampsia } \\
(n=47+4) \\
\end{array}$ & $41,55 \pm 2,63^{*}$ & $47,04 \pm 2,45^{*}$ & $56,16 \pm 2,72 *$ & $58,12 \pm 2,61 * * *$ & $60,24 \pm 2,01 * *$ & $62,16 \pm 2,41$ & $63,18 \pm 2,13$ \\
\hline & $\begin{array}{c}\text { Normal } \\
(n=55)\end{array}$ & $70,13 \pm 2,51$ & $\begin{array}{c}85,09 \pm 2,21 \\
\mathrm{p}<0,001\end{array}$ & $\begin{array}{c}79,32 \pm 2,19 \\
\mathrm{p}<0,05\end{array}$ & $\begin{array}{c}71,04 \pm 2,01 \\
\mathrm{p}<0,05\end{array}$ & $\begin{array}{c}67,62 \pm 2,23 \\
p<0,05\end{array}$ & $\begin{array}{c}66,83 \pm 2,92 \\
p>0,05\end{array}$ & $\begin{array}{c}65,94 \pm 2,44 \\
p>0,05\end{array}$ \\
\hline \multirow[t]{3}{*}{$\begin{array}{r}\text { The end-diastolic } \\
\text { volume of the left } \\
\text { ventricle (EDV), ml }\end{array}$} & $\begin{array}{c}\text { Mild PE } \\
(n=39)\end{array}$ & $101,19 \pm 3,01^{*}$ & $109,06 \pm 2,21 *$ & $109,32 \pm 2,19 *$ & $109,14 \pm 2,85$ & $106,14 \pm 2,45$ & $105,51 \pm 2,35$ & $104,66 \pm 2,48$ \\
\hline & $\begin{array}{c}\text { Severe } \\
\text { PE/eclampsia } \\
(n=47+4)\end{array}$ & $93,26 \pm 2,41^{*}$ & $102,07 \pm 2,21 *$ & $105,38 \pm 2,08 *$ & $105,36 \pm 2,83 * *$ & $105,01 \pm 3,15^{* *}$ & $105,52 \pm 2,15$ & $104,23 \pm 2,69$ \\
\hline & $\begin{array}{c}\text { Normal } \\
(n=55)\end{array}$ & $112,61 \pm 2,98$ & $\begin{array}{c}125,49 \pm 2,01 \\
\mathrm{p}<0,001\end{array}$ & $\begin{array}{c}120,18 \pm 2,01 \\
\mathrm{p}<0,05\end{array}$ & $\begin{array}{c}109,72 \pm 2,15 \\
\mathrm{p}<0,05\end{array}$ & $\begin{array}{c}105,20 \pm 2,01 \\
\mathrm{p}<0,01\end{array}$ & $\begin{array}{c}105,63 \pm 3,94 \\
p>0,05\end{array}$ & $\begin{array}{c}104,72 \pm 3,25 \\
\mathrm{p}>0,05\end{array}$ \\
\hline \multirow[t]{3}{*}{$\begin{array}{r}\text { Cardiac output }(\mathbf{C O}), 1 / \\
\min \end{array}$} & $\begin{array}{c}\text { Mild PE } \\
(n=39)\end{array}$ & $4,71 \pm 0,22 * *$ & $5,28 \pm 0,51 * *$ & $5,40 \pm 0,58 * *$ & $5,06 \pm 0,18 * *$ & $5,31 \pm 0,56$ & $5,02 \pm 0,58$ & $5,09 \pm 0,44$ \\
\hline & $\begin{array}{c}\text { Severe } \\
\text { PE/eclampsia } \\
(n=47+4)\end{array}$ & $4,06 \pm 0,31^{*}$ & $4,31 \pm 0,41^{*}$ & $5,01 \pm 0,38 * * *$ & $4,60 \pm 0,18^{* *}$ & $4,48 \pm 0,15^{* *}$ & $4,42 \pm 0,38$ & $4,44 \pm 0,29$ \\
\hline & $\begin{array}{c}\text { Normal } \\
(n=55)\end{array}$ & $5,51 \pm 0,28$ & $\begin{array}{c}6,87 \pm 0,21 \\
p<0,01\end{array}$ & $\begin{array}{c}6,08 \pm 0,22 \\
p<0,05\end{array}$ & $\begin{array}{c}5,44 \pm 0,18 \\
\mathrm{p}<0,05\end{array}$ & $\begin{array}{c}5,01 \pm 0,16 \\
\mathrm{p}<0,05\end{array}$ & $\begin{array}{c}5,02 \pm 0,31 \\
p>0,05\end{array}$ & $\begin{array}{c}5,13 \pm 0,28 \\
p>0,05\end{array}$ \\
\hline
\end{tabular}

